\title{
GMR
}

\section{Alleviation of streptozotocin-induced diabetes in nude mice by stem cells derived from human first trimester umbilical cord}

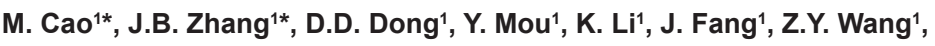 \\ C. Chen ${ }^{2}$, J. Zhao ${ }^{2 *}$ and S.M. Yie ${ }^{1}$ \\ ${ }^{1}$ Core Laboratory, School of Medicine, Sichuan Provincial People's Hospital \\ Affiliated to the University of Electronic Science and Technology of China, \\ Chengdu, China \\ ${ }^{2}$ Key Laboratory of Biological Resource and Ecological Environment of the Chinese \\ Education Ministry, College of Life Science, Sichuan University, Chengdu, China \\ *These authors contributed equally to this study. \\ Corresponding author: S.M. Yie \\ E-mail: 14857004@qq.com
}

Genet. Mol. Res. 14 (4): 12505-12519 (2015)

Received April 23, 2015

Accepted July 2, 2015

Published October 16, 2015

DOI http://dx.doi.org/10.4238/2015.October.16.18

\begin{abstract}
Cells isolated from human first trimester umbilical cord perivascular layer (hFTM-PV) tissues display the pluripotent characteristics of stem cells. In this study, we examined whether hFTM-PV cells can differentiate into islet-like clusters (ILCs) in vitro, and whether transplantation of the hFTM-PV cells with and without differentiation in vitro can alleviate diabetes in nude mice. The hFTM-PV cells were differentiated into ILCs in vitro through a simple stepwise culture protocol. To examine the in vivo effects of the cells, the hFTM-PV cells with and without differentiation in vitro were transplanted into the abdominal cavity of nude mice with streptozotocin (STZ)-induced diabetes. Blood glucose levels, body weight, and the survival probability of the diabetic nude mice were then statistically analyzed. The hFTM-PV cells were successfully induced into ILCs that could release insulin in response to elevated concentrations
\end{abstract}


of glucose in vitro. In transplantation experiments, we observed that mice transplanted with the undifferentiated hFTM-PV cells, embryonic body-like cell aggregations, or ILCs all demonstrated normalized hyperglycemia and showed improved survival rate compared with those without cell transplantation. The hFTM-PV cells have the ability to differentiate into ILCs in vitro and transplantations of undifferentiated and differentiated cells can alleviate STZ-induced diabetes in nude mice. This may offer a potential cell source for stem cell-based therapy for treating diabetes in the future.

Key words: Human first trimester umbilical cord; Stem cell; Diabetes

\section{INTRODUCTION}

Type I diabetes is a lifelong disease that is accompanied by hyperglycemia and results from the destruction of the insulin-producing beta cells of the pancreas. Standard treatment strategies for type I diabetes are based on different regimes of insulin injections with careful blood glucose monitoring. However, such treatment is frequently associated with severe hypoglycemic episodes because exogenous insulin cannot exactly mimic the physiology of insulin secretion (Efrat, 2008). While whole pancreas or islet transplantation is often considered a promising long-term solution for diabetes treatment, the benefits are limited by the difficulty of obtaining transplant materials and organ donations (Pileggi et al., 2006).

Therefore, generation of insulin-producing cells from various stem cell sources has become an important objective in the development of a cure for diabetes (Gangaram-Panday et al., 2007). Many studies have suggested that human embryonic stem cells (hESCs) (D'Amour et al., 2006), induced pluripotent stem cells (iPSCs) (Takahashi et al., 2007), and mesenchymal stem cells (MSCs) (Vija et al., 2009) may be possible cell sources for the generation of insulin-secreting cells. However, they have some problems associated with tumorigenicity, immunogenicity, etc. (Pappas and Yang, 2008; Sahu et al., 2009; Gutierrez-Aranda et al., 2010).

MSCs are multipotent stem cells that can differentiate into a variety of cell types including insulin-secreting cells, and can reduce blood glucose levels (Chao et al., 2008). One of the two important aspects of MSCs is that systemically administered MSCs home in on sites of ischemia or injury (Yagi et al., 2010). Therefore, it has also been reported that the transplantation of undifferentiated MSCs can reduce hyperglycemia in diabetic murines (Dong et al., 2008), as well as enhance all ogeneic islet engraftment in non-human primates (Berman et al., 2010), although the mechanisms by which this takes place remain undetermined. MSCs can be obtained from a variety of organs/tissues. In general, MSCs isolated from developmentally less mature tissues may be advantageous over those from mature sources (Baksh et al., 2007; Guillot et al., 2007).

We previously found that stem cells can be isolated from human first trimester umbilical cord perivascular layer (hFTM-PV) tissues. These hFTM-PV cells exhibit certain features of pluripotent stem cells that include expression of pluripotent stem cell markers and formation of embryoid body (EB)-like cell aggregations (Librach et al., 2009). A research group based in Canada has further discovered that hFTM-PV cells exhibit significantly greater proliferative potential and are more efficient in their in vitro differentiation towards selective mesenchymal cell types including chondrogenic and adipogenic lineages, as well as towards neuronal- and hepatocyte-like lineages when compared with TERM-PV cells (Hong et al., 2013).

Drawing on this evidence, we hypothesized that hFTM-PV cells are able to differentiate 
into insulin-secreting cells in vitro, and that the transplantation of hFTM-PV cells can alleviate diabetic hyperglycemia.

\section{MATERIAL AND METHODS}

\section{Isolation and culture of hFTM-PV cells}

Human first trimester umbilical cords were collected following spontaneous or therapeutic pregnancy interruptions after obtaining written consent from patients, which was approved by the Sichuan Provincial People's Hospital Institutional Review Board (IRB). To isolate umbilical cord stem cells, we followed a procedure outlined in the references (Librach et al., 2009; Hong et al., 2013).

\section{In vitro differentiation of ILCs from undifferentiated hFTM-PV cells}

Undifferentiated hFTM-PV cells were first cultured and expanded in $10 \%$ fetal bovine serum/ Dulbecco's modified Eagle medium (Gibco, Carlsbad, CA, USA) for 3-6 days in a 75-cm² culture flask. Next, the cells were transferred into ultra-low-attachment 6-well plates (Corning, USA) to form EB-like cell aggregations in an EB formation medium (Invitrogen, USA). After 7 days of culture, the cell aggregations were transferred to a $10-\mathrm{cm}^{2}$ culture plate with 1:1 DMEM/F12 medium and 1:100 insulin-transferrin-selenium (ITS), $1 \mathrm{mM}$ glutamine, and $5 \mu \mathrm{g} / \mathrm{mL}$ fibronectin (Roche, Germany) for 7 days. Subsequently, the cells were cultured in 1:1 DMEM/F12 with $10 \mathrm{ng} / \mathrm{mL}$ basic fibroblast growth factor (bFGF) (Gibco) for 7 days and with $10 \mathrm{mM}$ nicotinamide (Gibco) for another 5 days. Figure 1A summarizes the schematic procedure for the generation of ILCs from hFTM-PV cells.

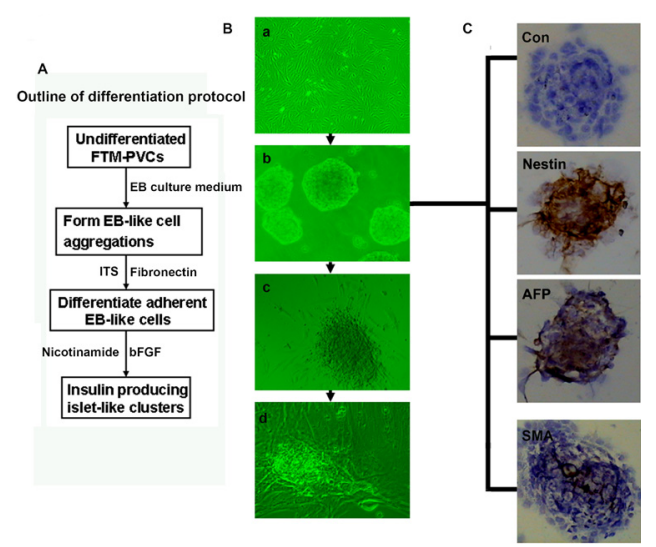

Figure 1. A. Schematic representation of the procedure for generating islet-like clusters from human first trimester umbilical cord perivascular layer ( $\mathrm{hFTM-PV)} \mathrm{cells.} \mathrm{The} \mathrm{differentiated} \mathrm{cells} \mathrm{were} \mathrm{transferred} \mathrm{into} \mathrm{ultra-low-attachment} \mathrm{six-}$ well plates to form embryonic body (EB)-like cell aggregations in an EB formation medium. Next, the cell aggregations were transferred into Dulbecco's modified Eagle medium (DMEM)/F12 islet maturation medium with insulin-transferrinselenium (ITS), glutamine, and fibronectin, and cultured on an adhesion plate for 7 days. Afterwards, the cells were cultured for 6-7 days in 1:1 DMEM/F12 with nicotinamide, glutamine, and basic fibroblast growth factor (bFGF) to form insulin-producing islet-like clusters, which were detected by immunocytochemical (ICC) staining (see Figure 2C). B. (a) The cell morphology of undifferentiated human hFTM-PV cells; (b) formation of EB-like cell aggregations; (c) differentiating aggregations; and (d) islet-like clusters (ILCs). C. The EB-like cell aggregations were characterized by the detection of markers of three germ lineages: alpha-fetoglobulin (AFP) for endoderm, nestin for ectoderm, and smooth muscle actin (SMA) for mesoderm. The scale bar is set to $100 \mu \mathrm{m}$. 


\section{Immunocytochemical staining}

The cultured hFTM-PV cells, EB-like cell aggregations, or ILCs were investigated by immunocytochemical staining. Source and dilution information for the primary antibodies is provided in Table 1. The cells were incubated with horseradish peroxidase (HRP)-conjugated goat anti-mouse IgG (1:1000) for $1 \mathrm{~h}$ at room temperature. Pancreases known to be positive for islet endocrine cell markers were used as positive controls. Negative controls for immunostaining were prepared by omission of the primary antibodies.

\begin{tabular}{llll}
\multicolumn{2}{c}{ Table 1. Source and dilution of primary antibodies used in this study. } \\
\hline Antibodies & Clone & Manufacturer & Dilution \\
\hline AFP & Rabbit polyclonal & Sigma-Aldrich & $1: 200$ \\
Nestin & Rabbit polyclonal & Millipore & $1: 200$ \\
SMA & Rabbit polyclonal & MyBioSource & $1: 200$ \\
C-peptide & Rabbit polyclonal & Millipore & $1: 200$ \\
Glucagon & Rabbit polyclonal & ZhongShanJinQiao & $1: 200$ \\
Insulin & Mouse monoclonal & ZhongShanJinQiao & $1: 200$ \\
HLA-G & Mouse monoclonal (HGY) & SichuanXinChuang & $1: 200$ \\
\hline
\end{tabular}

AFP = alpha-fetoglobulin; SMA = smooth muscle actin; HLA-G = human leukocyte antigen G.

\section{Reverse transcription polymerase chain reaction (RT-PCR)}

Total RNA was isolated from the hFTM-UC cells, EB-like cell aggregations, and ILCs. mRNA expression of $P D X 1, N G N 3, P A X 6$, and glyceraldehyde 3-phosphate dehydrogenase $(G A P G H)$ in the cells was determined by PCR using previously reported primer sequences and amplification protocols (Segev et al., 2004).

\section{Glucose-stimulated insulin release}

The hFTM-PV cell-derived ILCs were plated onto a 48-well plate in triplicate. The ILCs were first incubated in Roswell Park Memorial Institute (RPMI) 1640 medium containing $5 \mathrm{mM}$ glucose $\left(0.5 \mathrm{~mL}\right.$ per well) at $37^{\circ} \mathrm{C}$ for $3.5 \mathrm{~h}$. The supernatants were collected, and the same ILCs were further treated with $0.5 \mathrm{~mL}$ RPMI 1640 medium containing $25 \mathrm{mM}$ glucose and incubated at $37^{\circ} \mathrm{C}$ for another $3.5 \mathrm{~h}$. Finally, the supernatants were again collected for determination of insulin concentrations.

\section{Streptozotocin-induced diabetes in nude mice and cell transplantation therapy}

The Institutional Animal Care and Use Committees at the Sichuan Academy of Medical Sciences approved all animal procedures as protocol \#2010JY0043. Every effort was made to minimize animal suffering.

First, streptozotocin (STZ, Sigma) was intraperitoneally (ip) injected at $40 \mathrm{mg} / \mathrm{kg} / \mathrm{day}$ into 4-6-week-old male nude mice for 5 days to induce experimental diabetes. Blood glucose was measured by a glucometer (Accu-Chek, Roche Diagnostics, USA) from snipped tails. Only mice with stable blood glucose levels above $13 \mathrm{mM}$ after the STZ treatments were used in the transplantation experiments. We transferred the cells with and without differentiation of ILCs in vitro into the STZtreated nude mice to observe whether all the transplantations could alleviate type I diabetes. 
The animals were randomly divided into four groups that were ip injected with 1) undifferentiated hFTM-PV cells $(N=16), 2)$ EB-like cell aggregations $(N=11), 3)$ ILCs $(N=10)$, and $4)$ saline $(\mathrm{N}=8)$. Blood glucose levels were monitored every 3 days. At the same time, animals with healthy conditions and body weight were monitored until death or sacrificed for sample collection.

\section{Enzyme-linked immunosorbent assay (ELISA) for measuring insulin in culture- conditioned medium and serum}

Insulin production in both low- and high-glucose-treated ILC supernatants was analyzed using a human insulin-specific ELISA kit (Millipore, USA). Whole mouse blood samples were collected from the retro-orbital vein upon euthanasia, and serum was isolated by centrifugation. Serum insulin levels were simultaneously measured using a mouse insulin ELISA kit (Millipore, USA) and a human insulin-specific ELISA kit. All the analyses were performed according to the manufacturer instructions.

\section{Statistical analysis}

All statistical analyses were carried out using the SPSS software program (version 14), and the data are presented as mean \pm SEM. Statistical analysis of the data was performed by a one-way analysis of variance followed by Tukey's test. The Kaplan-Meier method was used to estimate the overall survival rate as a function of time. A P value of $<0.05$ was regarded as significant.

\section{RESULTS}

\section{Stem cell properties of hFTM-PV cells}

The primary isolates were heterogeneous, that is, most of the cells were spindle-shaped, resembling fibroblasts, and reached confluence every 5-7 days. After 2-3 passages, adherent cells comprised homogeneous cell layers with a fibroblasts-like morphology (Figure 1B-a). The cells consistently and strongly expressed OCT4, NANOG, SOX2, SSEA3, SSEA-4, TRA160, and TRA181 through passages 0 to 15, over 11 weeks of culture (Librach et al., 2009). The cells were able to form EB-like cell aggregations in the EB formation medium (Figure 1B-b). The cell aggregations expressed specific markers for three germ layers (alpha-fetoglobulin (AFP) for endoderm, nestin for ectoderm, and smooth muscle actin (SMA) for mesoderm cells (Sidhu et al., 2008), Figure $1 \mathrm{C}$ ), suggesting that the cells have the potential to differentiate into various types of cell raised from these three germ layers.

\section{Differentiation of hFTM-PV cells into insulin-secreting ILCs in vitro}

Although the hFTM-PV cells per se are mainly a mesenchymal lineage with pericyte-like properties (Hong et al., 2013), the cells can form EB-like aggregations in which a large number of cells can express the endodermal marker AFP (Figure 1C). We modified a differentiation protocol without the steps of activin A and retinoic acid treatment, and used it to differentiate ES cells into definitive endoderm and pancreatic fates (Jiang et al., 2007; Mayhew and Wells, 2010). Furthermore, in our protocol, no N2/B27 supplements, which are used to enrich nestin-positive cells (Lumelsky et al., 2001), were used. However, both bFGF, which acts to expand pancreatic progenitors and/ 
or promote a pancreatic fate, and nicotinamide, which acts as a late-stage 'maturation' factor in DMEM/F12 (Ohgawara et al., 1993; Otonkoski et al., 1993) were included in our protocol. Under differentiation conditions, ILCs can be formed following the formation of EB-like cell aggregations and induction of differentiation/maturation (Figure 1B-c and -d).

RT-PCR analysis (Figure 2A) confirmed the expression of pancreatic and duodenal homeobox-1 (PDX1), which encodes for a transcription factor that is necessary for pancreatic development (Zhou et al., 2008), neurogenin-3 (NGN3), basic helix-loop-helix (bHLH) transcription factor, which is critical for the specification of endocrine cells in the pancreatic islets (Villasenor et al., 2008), and homeodomain transcription factor PAX6, which is an important factor in pancreatic islet beta-cell development (Schisler et al., 2008), in the undifferentiated hFTM-PV cells, EB-like cell aggregations, and ILCs. However, the expression of PDX1 and PAX6 was significantly higher in ILCs than in the hFTM-PV cells and EB-like cell aggregations. Additionally, expression of NGN3 was higher in hFTM-PV cells and EB-like cell aggregations compared with ILCs (Figure 2B).

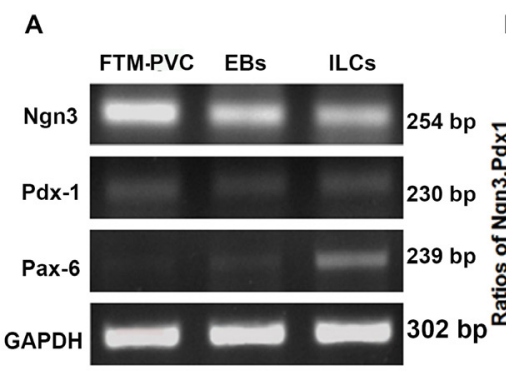

$$
\text { B }
$$

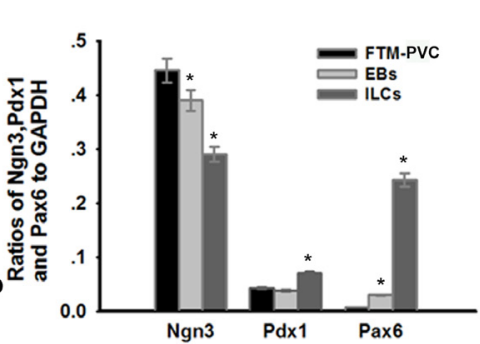

C

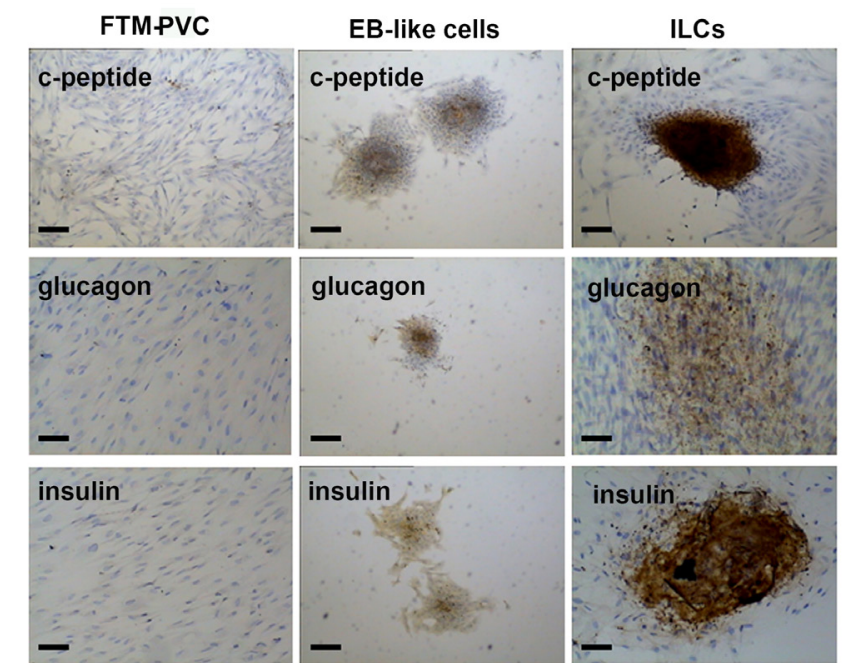

Figure 2. A. Reverse transcription polymerase chain reaction (RT-PCR) analysis of PDX1, NGN3, and PAX6 expression during the islet differentiation in which glyceraldehyde 3-phosphate dehydrogenase (GAPDH) was used as a reference gene. PCR products were separated by gel electrophoresis. B. Relative abundance of the gene transcripts. *Indicates significant $(P<0.05)$ differences in transcript levels during the differentiation. C. Immunocytochemical analysis of the expression of islet endocrine cell markers c-peptide, glucagon, and insulin in the first trimester umbilical cord perivascular layer (FTM-PV) cells, embryonic body (EB)-like cell aggregations, and islet-like clusters (ILCs). The scale bar is set to $100 \mu \mathrm{m}$. 
Immunohistochemical staining was performed to investigate the expression of c-peptide, glucagon, and insulin in undifferentiated hFTM-PV cells, EB-like cell aggregations, and ILCs. Data from one representative experiment are presented in Figure $2 \mathrm{C}$. The undifferentiated FTM-PV cells did not express c-peptide, glucagon, or insulin (Figure 2C, column 1). The EB-like cell aggregations showed weak but detectable expression of c-peptide, glucagon, and insulin (Figure 2C, column 2). The cells in mature ILCs strongly expressed all markers (Figure 2C, panel 3), suggesting that these cells represent an endocrine cell type similar to islets in the pancreas.

The hFTM-PV cell-derived ILC's ability to secrete insulin in response to glucose stimulation was tested in the next experiment. Here, ILCs derived from hFTM-PV cells released insulin in a glucose-dependent manner (Figure 3), while the undifferentiated cells and the EB-like cell aggregations did not release a measurable amount of insulin into the medium (data not show). Five separate ILC preparations were subjected to a glucose-stimulated insulin release analysis. The average insulin concentration in a medium containing $25 \mathrm{mM}$ glucose was $23.2 \pm 5.3 \mathrm{mlU}$ (mean $\pm \mathrm{SE}$ ) and the average insulin concentration in a medium containing $5 \mathrm{mM}$ glucose was $7.0 \pm 0.5$ $\mathrm{mIU}$ (mean $\pm \mathrm{SE}$ ). Comparing these results, $\mathrm{P}=0.002$, which indicates that the ILCs generated by our protocol were able to secrete insulin in response to high concentrations of glucose.

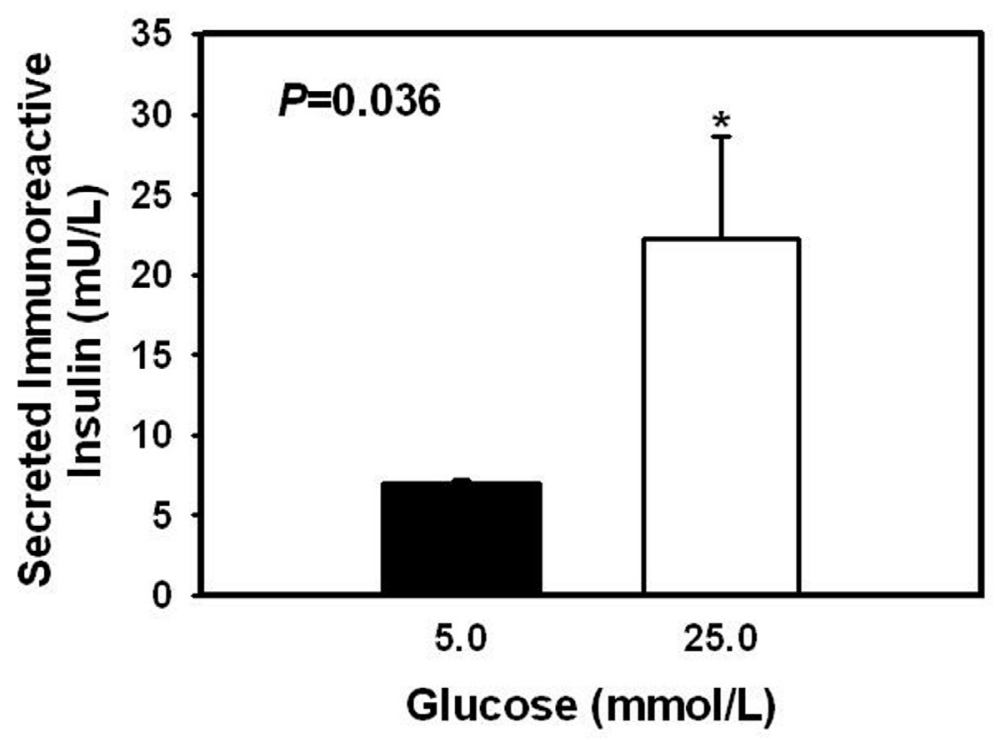

Figure 3. The islet-like cell clusters (ILCs) released insulin in response to glucose in vitro. After the induction of ILCs, the amount of insulin in the culture media was measured by an enzyme-linked immunosorbent assay (ELISA) after the cells had been exposed to low or high concentrations of glucose. The Student's t-test showed significant differences between the groups $(P<0.05)$. The asterisk denotes a significant difference between the two groups.

\section{Effect of transplantation in vivo}

Normal blood glucose levels in nude mice ranged from 3.8 to $7.5 \mathrm{mM}$ with a mean value of $5.5 \mathrm{mM}$. The blood glucose levels were monitored every 3 days. After STZ treatment, the blood glucose levels dramatically increased to $16.4 \pm 2.9 \mathrm{mM}$ (mean $\pm \mathrm{SE}$ ) and continued to rise until the animals died. At the same time, animals not subjected to STZ treatment had normal blood glucose levels during the entire experimental period (Figure 4A). 
A
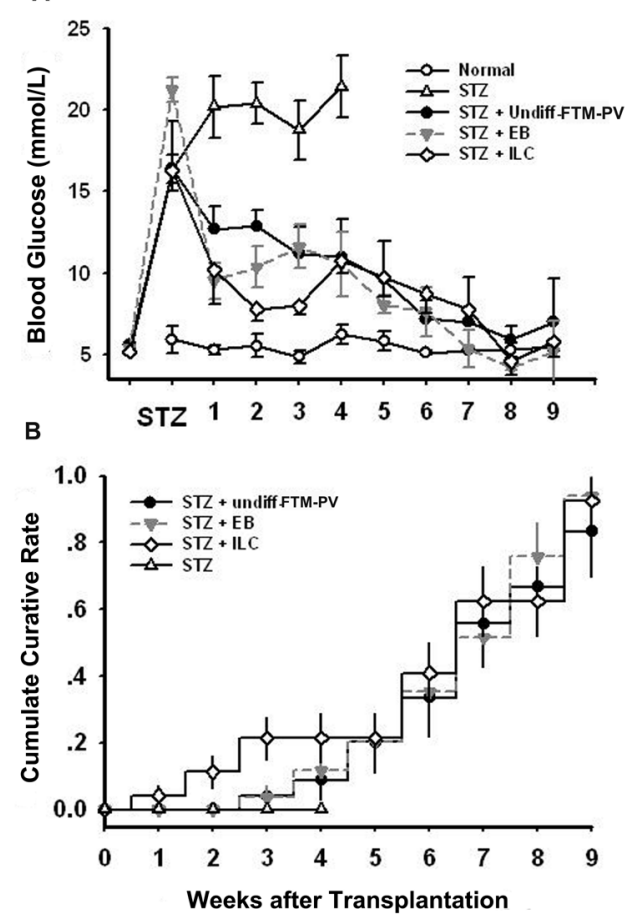

Figure 4. Effects on blood glucose levels of the transplantation of human first trimester umbilical cord perivascular layer (hFTM-PV) cells with and without differentiation in vitro. A. Statistical analysis revealed that the transplantations significantly alleviated diabetic hyperglycemia $(F=3.14, P=0.009$ for the transplantation of undifferentiated FTM-PV cells, $F=15.6, P=0.0001$ for the transplantation of embryonic body $(E B)$-like cell aggregations and $F=6.59, P=$ 0.0001 for islet-like cell clusters (ILCs), respectively). B. Cumulative curative effect of transplantation of hFTM-PV cells with and without differentiation in vitro on hyperglycemia in nude mice with streptozotocin (STZ)-induced diabetes. There was no significant difference among the three groups.

In the ILC transplantation group, the average blood glucose levels were $16.3 \pm 1.24 \mathrm{mM}$ before transplantation. After transplantation, hyperglycemia gradually decreased $(F=6.59, P=$ 0.0001 , Figure $4 \mathrm{~A})$. By the second week after transplantation, the blood glucose levels had been revised to near normal ranges $(7.81 \pm 0.74 \mathrm{mM})$, although there were slight increasing fluctuations between the fourth and sixth weeks. Transplantation of the undifferentiated cells also progressively alleviated hyperglycemia, starting from 3-7 days after transplantation until the end of the experiments $(F=3.14, P=0.009$, Figure $4 A)$. In this group, initial blood glucose levels were $16.4 \pm 2.9 \mathrm{mM}$ after STZ treatment. At the sixth week of transplantation, the blood glucose concentrations in the animals reached normal ranges $(7.22 \pm 2.74 \mathrm{mM})$. In the EB-like cell aggregations transplantation group, STZ induced hyperglycemia with an average level of $21.2 \pm 0.73 \mathrm{mM}$ blood glucose concentration. Similar to the ILCs group, the transplantation of EB-like cell aggregations dramatically decreased blood glucose levels in the first transplantation week by $53.8 \%$ from initial hyperglycemia. The decrease continued and reached normal ranges of blood glucose levels $(7.6 \pm 1.48 \mathrm{mM})$ at the sixth week $(F=15.6, P=0.0001$, Figure $4 A)$.

There was no significant difference in terms of cumulative curative effect among the three groups by a log-rank analysis $\left(X^{2}=0.908, P=0.635\right.$, Figure $\left.4 B\right)$. These results 
demonstrate that all types of transplantation are able to ease hyperglycemia induced by STZ treatment for more than 2 months.

In addition, glucose tolerance tests were performed after the sixth week of transplantation, and showed that blood glucose levels of both normal and cell-treated mice dropped to initial levels within 150 min (data not show).

The body weight of the mice at Weeks 0 and 1 after transplantation was similar in all groups. The weight of diabetic mice treated with saline was significantly lower than those with the three types of transplantation after 2 weeks $(P=0.047)$. The body weight of diabetic mice treated with saline was further reduced before death (around 4 weeks), and was also significantly lower than in those that had received the three types of transplantation before death (around 9 weeks) $(P=0.001)$. Although body weight in the transplantation groups also decreased gradually compared with before the diabetes inductions, no significant differences in body weight changes were observed among the three transplantation groups (Figure 5).

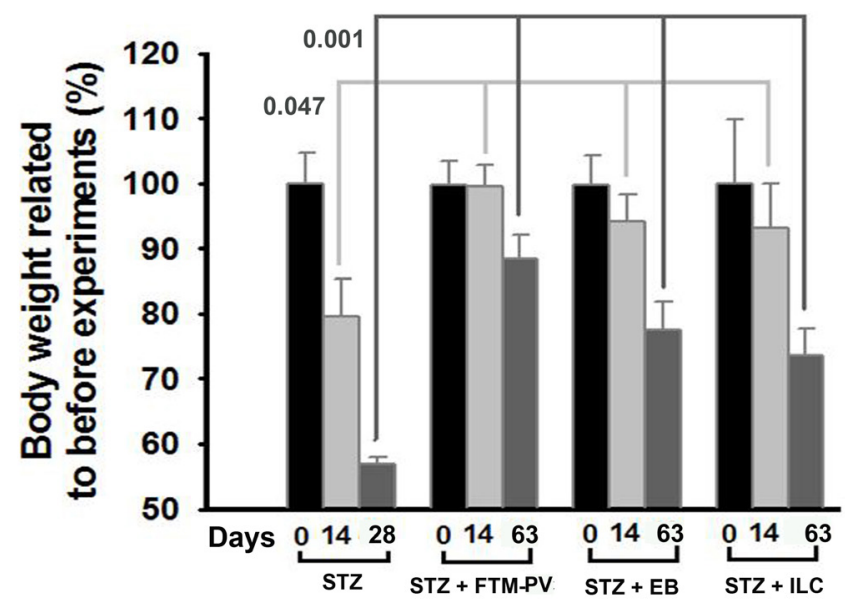

Figure 5. Effects of the transplantation of human first trimester umbilical cord perivascular layer (hFTM-PV) cells with and without differentiation in vitro on the body weight of nude mice with streptozotocin (STZ)-induced diabetes. The gray line indicates significant differences of body weight among the treatment groups after 14 days $(P=0.047)$, and the dark line indicates significant differences in body weight among the treatment groups before death $(P=0.001)$.

Moreover, serum mouse insulin levels were less than $0.2 \mathrm{ng} / \mathrm{mL}$ with an average of 0.102 $\mathrm{ng} / \mathrm{mL}$ in the mice with STZ-induced diabetes. In the mice with STZ-induced diabetes treated with cell transplantations, serum mouse insulin levels were still less than $0.2 \mathrm{ng} / \mathrm{mL}$ with an average of $0.119 \mathrm{ng} / \mathrm{mL}$. In contrast, human serum insulin levels were $5.70 \pm 0.31,5.56 \pm 0.06$, and $5.18 \pm$ $0.42 \mathrm{mU} / \mathrm{L}$ in the groups with undifferentiated FTM-PV cells, EB-like cell aggregations, and ILCs, respectively. These results suggest that the effect of transplantation on hyperglycemia in vivo is mainly exerted by human insulin, which may be secreted by donor cells.

The mice in each group were observed for their survival periods. All the diabetic mice without cell transplantations died 4 weeks after the STZ treatment, while most of the diabetic mice with cell transplantations survived for more than 9 weeks $(N=9,6$, and 6 for the transplantation groups with undifferentiated FTM-PV cells, EB-like cell aggregations, and ILCs, respectively). The data shown in Figure 6A indicate that the survival ratio in mice with STZ-induced diabetes was significantly lower compared with diabetic mice with cell transplantations $(P<0.05)$, and the 
survival ratio of the undifferentiated cell group at 3 weeks after transplantation was lower than in the EB-like cell aggregations and ILCs groups $(P<0.05)$. After 3 weeks, however, the survival ratio in the undifferentiated cell group remained until 9 weeks and showed no significant difference from the EB-like cell aggregations and ILC groups. Further, in the entire cohort, the overall survival rates of the diabetic mice with cell transplantations were significantly higher compared with those mice that had not received cell transplantations $(P=0.005$, log-rank test, Figure $6 B)$. However, there was no significant difference among the cell treatment groups.

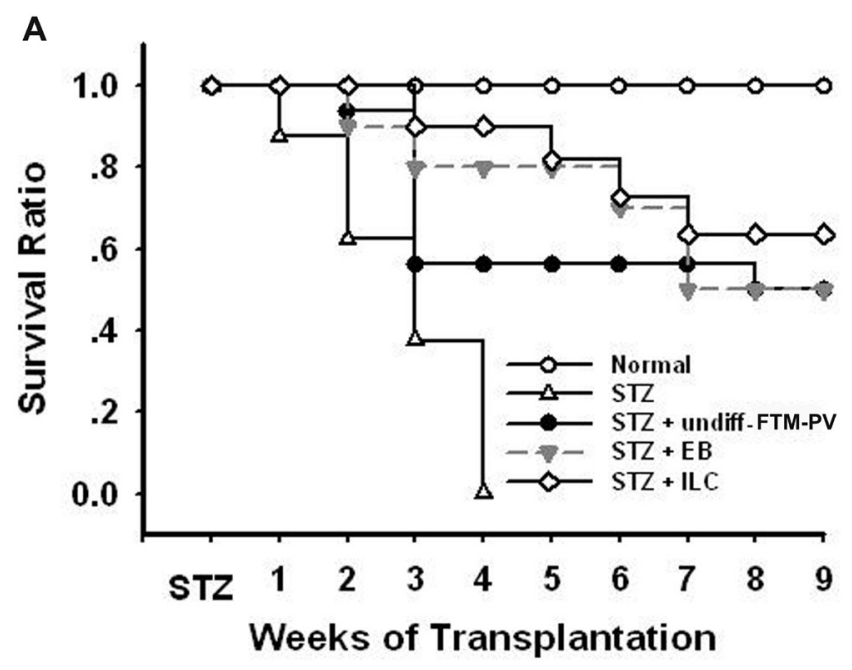

B

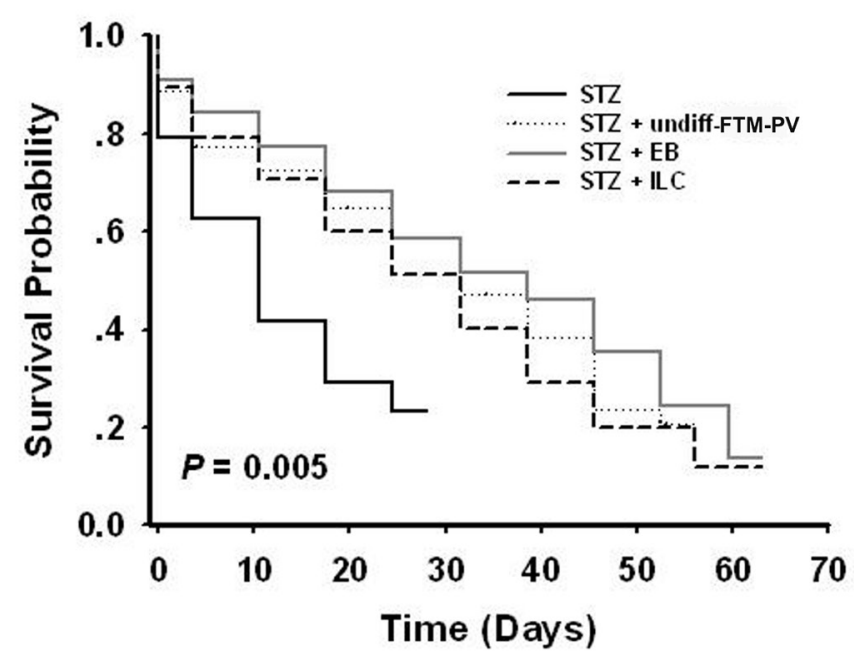

Figure 6. Effects of the transplantation of human first trimester umbilical cord perivascular layer (hFTM-PV) cells with and without differentiation in vitro on the survival ratio $(\mathbf{A})$, and the survival probability $(\mathbf{B})$ of nude mice with streptozotocin (STZ)-induced diabetes. In the entire cohort, the overall survival rates of the diabetic mice with cell transplantations were significantly higher compared with those without cell transplantations $(P=0.005$, log-rank test). However, there was no significant difference among the cell treatment groups. 


\section{HLA-G is expressed in undifferentiated hFTM-PV cells, EB-like cell aggregations, and ILCs}

Since MSCs possess potent immunoregulatory properties (Gebler et al., 2012), and human leukocyte antigen $G$ (HLA-G) is a key molecule of the MSC immunoregulatory function (Selmani et al., 2009), we performed immunocytochemical staining with an anti-HLA-G-specific antibody (HGY) that specifically recognizes HLA-G1 and its soluble counterpart HLA-G5 (Yie et al., 2007; Cao et al., 2011) to investigate the expression of HLA-G proteins in the hFTM-PV cells, EBlike cell aggregations, and ILCs. HLA-G expression was detected in all three types of cell (Figure 7). Interestingly, the expression of HLA-G was detected in the ILCs but not in the surrounding cells (Figure 7), which suggests that HLA-G is a determinant of the HLA repertoire in the human pancreas that is uniquely restricted to the endocrine cells.

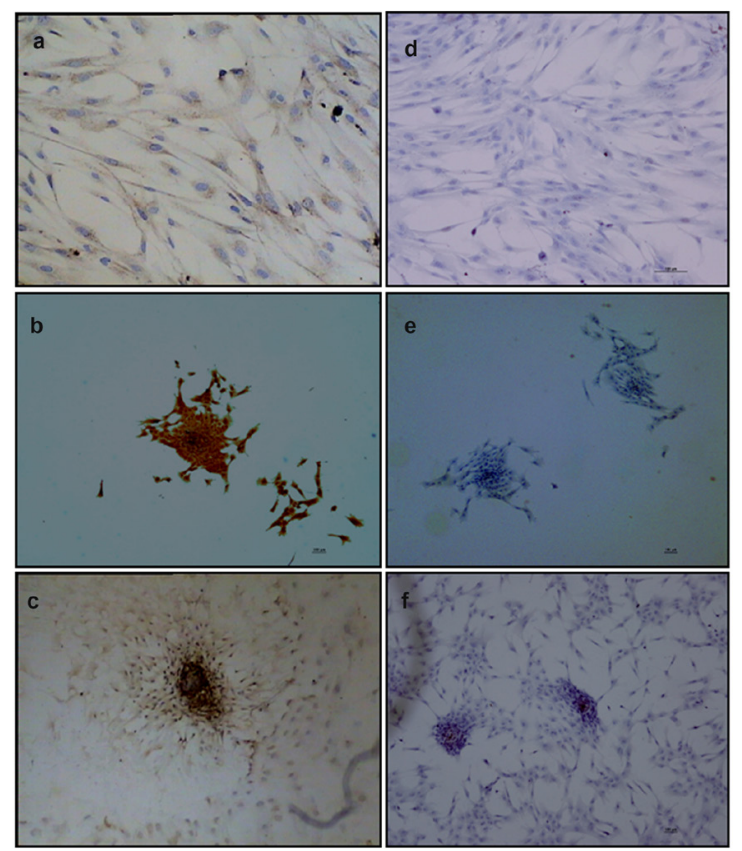

Figure 7. HLA-G protein expression levels in undifferentiated human first trimester umbilical cord perivascular layer (hFTM-PV) cells (a), embryonic body (EB)-like aggregations (b) formed from hFTM-PV cells, and the islet-like clusters (ILCs) (c) induced from EB-like cell aggregations were detected by immunohistochemical analysis. Negative controls for the immune staining were performed by omission of the primary antibodies (d-f). Original magnification: 40X.

\section{DISCUSSION}

It has been unequivocally established that the pancreas arises from the endoderm (Edlund, 2002), and, physiologically, $\beta$-cell development can be broken down into four steps. Based on this understanding of the process of pancreas development, a stepwise process that recapitulates all the major stages of $\beta$-cell development has been successfully used to direct ESCs to differentiate into endoderm-derived, mature, and glucose-responsive $\beta$-cells (Mayhew and Wells, 2010).

hFTM-PV cells can be grown using low-attachment dishes in the presence of a complete 
growth medium that lacks leukemia inhibitory factor, and they have the ability to form EB-like cell aggregations that express markers for the endoderm, ectoderm, and mesoderm (Librach et al., 2009). However, it is unclear how the cells are converted to their original status under these conditions. Perhaps this is one of the reasons that the cells keep expressing high levels of pluripotent stem cell-associated transcripts and/or proteins such as OCT4, NANOG, SOX-2, SSEA-3, SSEA-4, TRA-1-60, and TRA-1-81 (Librach et al., 2009). Nevertheless, the differentiation protocol used in the study starts from the EB-like cell aggregations, and is similar to the first stage of endoderm formation in $\beta$-cell development from ESCs (Mayhew and Wells, 2010).

Pancreatic development involves the proliferation of pancreatic progenitors and their segregation into either exocrine or endocrine cell types. The developmental process is controlled by a number of signal pathways, in which PDX1, NGN3, and PAX6 may play a critical role (Schisler et al., 2008; Rukstalis and Habener, 2009). In the present study, the transcripts of the PDX1 and $P A X 6$ genes increased while the transcript of the NGN3 gene decreased from the undifferentiated hFTM-PV cells and EB-like cell aggregations to the ILCs. This suggests that the cell source per se contained pancreatic development and endocrine cell development cell populations, and that our simple stepwise differentiation protocol can efficiently differentiate the cells into ILCs in vitro.

After the EB-like cell aggregations were re-cultured in non-EB formation media, the cells were changed into islet cells in the medium containing high glucose, high insulin, nicotinamide, and bFGF. At the ILC stage, the clusters strongly expressed c-peptide, glucagon, and insulin, implying that the cell clusters comprised pancreatic endocrine cells. The glucose-stimulated insulin release test showed a response to high concentrations of glucose, which further implies that these insulinproducing cells were functional and mature islet endocrine cells. Moreover, transplantation of the ILCs increased general health and reversed hyperglycemia. The survival time of nude mice with STZ-induced diabetes also demonstrated the functionality and maturity of the clusters in vivo.

The transplantation of undifferentiated MSCs in the Wharton's jelly of human third trimester umbilical cord had no effect on the alleviation of hyperglycemia, and decreased the body weight and survival ratio in rats with STZ-induced diabetes (Chao et al., 2008). However, in the current study, the transplantation of undifferentiated hFTM-PV cells or EB-like cell aggregations was able to alleviate diabetic conditions by providing insulin synthesized by the donor cells. This suggests that the hFTM-PV cells may have more plasticity than MSCs from first trimester umbilical cord in terms of alleviating diabetes.

However, our results are similar but not identical to those of previous studies reported by Ezquer et al. and Dong et al. (2008), in which MSC transplantations lowered the blood glucose levels of the recipients by supporting the regeneration of recipient $\beta$-cells rather than providing insulin synthesized by the donor cells. Unfortunately, in the present study, we injected the cells into the peritoneal cavity of the animals rather than transplanting the cells into the liver or kidney. As a result, we could not determine whether the undifferentiated cells and EB-like cell aggregations were differentiated into $\beta$-cells under in vivo diabetic conditions or determine the status of the ILCs. Therefore, in future studies we are planning to investigate the optimal anatomical sites for cell transplantation to enhance the survival and function of the ILCs, and determine the fate of undifferentiated cells in vivo.

It has been demonstrated that MSCs possess potent immunoregulatory properties that could be exploited to suppress allograft rejection following transplantation (Vija et al., 2009; Gebler et al., 2012). This is because MSCs have the ability to suppress T-cell responses via various immunomodulatory pathways including the production of the immunomodulatory cytokines TGF- $\beta$ and IL-10, as well as enzymatic pathways involving indoleamine 2,3-dioxygenase, nitric oxide synthase, and heme oxygenase-1 (Brusko, 2009; Vija et al., 2009; Gebler et al., 2012). 
In addition, HLA-G, a non-classical MHC class lb molecule, has been reported to be expressed in hES cells and MSCs isolated from either fetal or adult tissues (Weiss et al., 2008; Giuliani et al., 2011; Verloes et al., 2011). HLA-G is characterized by low polymorphism, restricted tissue distribution, and immune tolerogenic functions that may contribute to avoiding serious complications arising from alloimmune rejections in stem cell-based therapy (Gebler et al., 2012; Soleymaninejadian et al., 2012).

Fetal liver derived-MSCs display remarkably longer-lasting immunomodulatory properties compared with adult BM-MSCs, and are more efficient at inducing T-cell apoptosis and secretion of the immunosuppressive cytokine IL-10 (Cirulli et al., 2006). Interestingly, it has been reported that HLA-G is constitutively expressed in the endocrine compartment of the human pancreas, which may have potentially important implications for the progression of autoimmunity as well as for the establishment of transplant tolerance in this tissue (Cirulli et al., 2006). Consistent with these reports, our results showed that HLA-G is not only expressed in undifferentiated FTM-PV cells and EB-like cell aggregations, but can also be detected in ISLs restricted to the endocrine cells.

Given the properties of hFTM-PV cells, we hypothesized that they are a favorable source of stem cells for conversion into insulin-producing cells owing to their greater proliferative potential and higher differentiation efficiency in vitro (Librach et al., 2009; Hong et al., 2013), their greater capacity to alleviate diabetes, and the lower risk of rejection they offer compared with other sources of MSCs.

In conclusion, in this study, we demonstrated that hFTM-PV cells are able to differentiate into mature ISLs through EB-like cell aggregations under in vitro culture conditions. Moreover, transplantation of the cells with or without differentiation in vitro can alleviate STZ-induced diabetes in nude mice. Since younger sources of adult stem cell populations show a greater proliferative potential and plasticity than their older counterparts (Librach et al., 2009; Choumerianou et al., 2010; Hong et al., 2013), hFTM-PV cells may have more potential as a starting material for the generation of an unlimited supply of insulin-secreting cells.

\section{Conflicts of interest}

The authors declare no conflict of interest.

\section{ACKNOWLEDGMENTS}

Research financially supported by the Sichuan Provincial Research Program for Distinguished Young Scientists (\#2011JQ0018), the National Natural Science Foundation of China, the Program for New Century Excellent Talent in University, and the Fundamental Research Funds for the Central Universities (\#81301872, \#31270175, \#NCET-13-0397, \#2013SCU04B14).

\section{REFERENCES}

Baksh D, Yao R and Tuan RS. (2007). Comparison of proliferative and multilineage differentiation potential of human mesenchymal stem cells derived from umbilical cord and bone marrow. Stem Cells. 25: 1384-1392.

Berman DM, Willman MA, Han D, Kleiner G, et al. (2010). Mesenchymal stem cells enhance allogeneic islet engraftment in nonhuman primates. Diabetes 59: 2558-2568.

Brusko TM (2009). Mesenchymal stem cells: a potential border patrol for transplanted islets? Diabetes 58: 1728-1729.

Cao M, Yie SM, Liu J, Ye SR, et al. (2011). Plasma soluble HLA-G is a potential biomarker for diagnosis of colorectal, gastric, esophageal and lung cancer. Tissue Antigens 78: 120-128.

Chao KC, Chao KF, Fu YS and Liu SH (2008). Islet-like clusters derived from mesenchymal stem cells in Wharton's Jelly of the human umbilical cord for transplantation to control type 1 diabetes. PLoS One 3: e1451. 
Choumerianou DM, Martimianaki G, Stiakaki E, Kalmanti L, et al. (2010). Comparative study of stemness characteristics of mesenchymal cells from bone marrow of children and adults. Cytotherapy 12: 881-887.

Cirulli V, Zalatan J, McMaster M, Prinsen R, et al. (2006). The class I HLA repertoire of pancreatic islets comprises the nonclassical class Ib antigen HLA-G. Diabetes 55: 1214-1222.

D'Amour KA, Bang AG, Eliazer S, Kelly OG, et al. (2006). Production of pancreatic hormone-expressing endocrine cells from human embryonic stem cells. Nat. Biotechnol. 24: 1392-1401.

Dong QY, Chen L, Gao GQ, Wang L, et al. (2008). Allogeneic diabetic mesenchymal stem cells transplantation in streptozotocininduced diabetic rat. Clin. Invest. Med. 31: E328-E337.

Edlund H (2002). Pancreatic organogenesis - developmental mechanisms and implications for therapy. Nat. Rev. Genet. 3: 524-532.

Efrat S (2008). Beta-cell replacement for insulin-dependent diabetes mellitus. Adv. Drug Deliv. Rev. 60: 114-123.

Ezquer FE, Ezquer ME, Parrau DB, Carpio D, et al. (2008). Systemic administration of multipotent mesenchymal stromal cells reverts hyperglycemia and prevents nephropathy in type 1 diabetic mice. Biol Blood Marrow Transplant. 14:631-40.

Gangaram-Panday ST, Faas MM and de Vos P (2007). Towards stem-cell therapy in the endocrine pancreas. Trends Mol. Med. 13: 164-173.

Gebler A, Zabel O and Seliger B (2012). The immunomodulatory capacity of mesenchymal stem cells. Trends Mol. Med. 18: 128-134.

Giuliani M, Fleury M, Vernochet A, Ketroussi F, et al. (2011). Long-lasting inhibitory effects of fetal liver mesenchymal stem cells on T-lymphocyte proliferation. PLoS One. 6: e19988.

Guillot PV, Gotherstrom C, Chan J, Kurata H, et al. (2007). Human first-trimester fetal MSC express pluripotency markers and grow faster and have longer telomeres than adult MSC. Stem Cells 25: 646-654.

Gutierrez-Aranda I, Ramos-Mejia V, Bueno C, Munoz-Lopez M, et al. (2010). Human induced pluripotent stem cells develop teratoma more efficiently and faster than human embryonic stem cells regardless the site of injection. Stem Cells 28: 1568-1570.

Hong SH, Maghen L, Kenigsberg S, Teichert AM, et al. (2013). Ontogeny of human umbilical cord perivascular cells: molecular and fate potential changes during gestation. Stem Cells Dev. 22: 2425-2439.

Jiang W, Shi Y, Zhao D, Chen S, et al. (2007). In vitro derivation of functional insulin-producing cells from human embryonic stem cells. Cell Res. 17: 333-344.

Librach CL, Yie SM and Xiao R (2009). Method of Isolation and Use of Cells Derived From First Trimester Umbilical Cord Tissue United States Patent Application 20090074731. http://www.freepatentsonline.com/y2009/0074731.html. Accessed June 16, 2015.

Lumelsky N, Blondel O, Laeng P, Velasco I, et al. (2001). Differentiation of embryonic stem cells to insulin-secreting structures similar to pancreatic islets. Science 292: 1389-1394.

Mayhew CN and Wells JM (2010). Converting human pluripotent stem cells into beta-cells: recent advances and future challenges. Curr. Opin. Organ Transplant. 15: 54-60.

Ongawara H, Kawamura M, Honda M, Karibe S, et al. (1993). Reversal of glucose insensitivity of pancreatic B-cells due to prolonged exposure to high glucose in culture: effect of nicotinamide on pancreatic B-cells. Tohoku J. Exp. Med. 169: 159-166.

Otonkoski T, Beattie GM, Mally MI, Ricordi C, et al. (1993). Nicotinamide is a potent inducer of endocrine differentiation in cultured human fetal pancreatic cells. J. Clin. Invest. 92: 1459-1466.

Pappas JJ and Yang (2008). Human ESC vs. iPSC-pros and cons. J. Cardiovasc. Transl. Res. 1: 96-99.

Rukstalis JM and Habener JF (2009). Neurogenin3: a master regulator of pancreatic islet differentiation and regeneration. Islets 1: 177-184.

Sahu S, Tosh D and Hardikar AA (2009). New sources of beta-cells for treating diabetes. J. Endocrinol. 202: 13-16.

Schisler JC, Fueger PT, Babu DA, Hohmeier HE, et al. (2008). Stimulation of human and rat islet beta-cell proliferation with retention of function by the homeodomain transcription factor Nkx6.1. Mol. Cell. Biol. 28: 3465-3476.

Segev H, Fishman B, Ziskind A, Shulman M, et al. (2004). Differentiation of human embryonic stem cells into insulin-producing clusters. Stem Cells 22: 265-274.

Selmani Z, Naji A, Gaiffe E, Obert L, et al. (2009). HLA-G is a crucial immunosuppressive molecule secreted by adult human mesenchymal stem cells. Transplantation 87: S62-S66.

Sidhu KS, Ryan JP and Tuch BE. (2008). Derivation of a new human embryonic stem cell line, endeavour-1, and its clonal propagation. Stem Cells Dev. 17:41-51.

Soleymaninejadian E, Pramanik K and Samadian E (2012). Immunomodulatory properties of mesenchymal stem cells: cytokines and factors. Am. J. Reprod. Immunol. 67: 1-8.

Takahashi K, Tanabe K, Ohnuki M, Narita M, et al. (2007). Induction of pluripotent stem cells from adult human fibroblasts by defined factors. Cell 131: 861-872. 
Verloes A, Van de Velde H, LeMaoult J, Mateizel I, et al. (2011). HLA-G expression in human embryonic stem cells and preimplantation embryos. J. Immunol. 186: 2663-2671.

Vija L, Farge D, Gautier JF, Vexiau P, et al. (2009). Mesenchymal stem cells: Stem cell therapy perspectives for type 1 diabetes. Diabetes Metab. 35: 85-93.

Villasenor A, Chong DC and Cleaver O (2008). Biphasic Ngn3 expression in the developing pancreas. Dev. Dyn. 237: 32703279.

Weiss ML, Anderson C, Medicetty S, Seshareddy KB, et al. (2008). Immune properties of human umbilical cord Wharton's jelly-derived cells. Stem Cells 26: 2865-2874.

Yagi H, Soto-Gutierrez A, Parekkadan B, Kitagawa Y, et al. (2010). Mesenchymal stem cells: Mechanisms of immunomodulation and homing. Cell Transplant. 19: 667-679.

Yie SM, Yang H, Ye SR, Li K, et al. (2007). Expression of human leukocyte antigen G (HLA-G) correlates with poor prognosis in gastric carcinoma. Ann. Surg. Oncol. 14: 2721-2729.

Zhou Q, Brown J, Kanarek A, Rajagopal J, et al. (2008). In vivo reprogramming of adult pancreatic exocrine cells to beta-cells. Nature 455: 627-632. 